\title{
Neuro-critical care versus general critical care for neurological injury: Beneficial evidence
}

\author{
Ian Tweedie ${ }^{1,2}$
}

\section{INTRODUCTION}

In many parts of the world, patients with neurological injury requiring critical care are managed in one of three models of intensive care; General Intensive Care Units (GICU) without direct neurosurgical/neurological input, ICUs co-located with a neuroscience unit, which may be a mixed speciality GICU with direct input from neuroscience specialists or a stand-alone Neuro-ICU (NICU) embedded within a neuroscience unit. Thus, it would be useful to start with a definition of neuro-critical care as opposed to critical care. Smith and Menon ${ }^{[1]}$ provide a good definition in their chapter in the new Guide to the Provision of a Critical Care Service (GPICS) document from the Intensive Care Society:

"Neuro-critical care is devoted to the comprehensive care of critically ill patients with neurological or neurosurgical disease. Care of such patients requires an understanding of the physiology and pathophysiology common to brain diseases in general, as well as the skills and knowledge to treat a range of specific conditions. Given the exquisite vulnerability of the injured brain to physiological insults, optimal care of such patients also demands meticulous attention to maintenance of systemic and cerebral physiological targets while ensuring appropriate protection of extra-cranial organs."

In 2004 Smith $^{[2]}$ asked the question "Neuro-critical care: Has it come of age?" In his conclusion, he noted that available data suggest that outcomes in neuro-specific ICUs might be better than in GICUs. He also states that we need to demonstrate this conclusively and show why. More than 10 years later can we answer this question

'Walton Centre, Liverpool, UK, '2Immediate Past President, Neuroanaesthesia and Critical Care Society of Great Britain and Ireland

\begin{tabular}{|l|l|}
\hline \multicolumn{2}{|c|}{ Access this article online } \\
\hline Quick Response Code: & Website: \\
\hline & www.jnaccjournal.org \\
\cline { 1 - 2 } & \\
\hline & DOI: \\
\hline
\end{tabular}

more conclusively and for all areas of neurologic injury? Kramer and Zygun in 2011 ${ }^{[3]}$ and again in $2014^{[4]}$ asked similar questions. Both times, they concluded that the body of evidence is strongly supporting the case for better outcomes but that there is still much to understand as to the how and why. In 2015, Smith and Menon briefly review the evidence in the GPICS ${ }^{[1]}$ document and comment that it now seems clear that the care does not necessarily need to be in a single speciality unit NICU, but more importantly that multidisciplinary expertise in the care of the sick brain is available directly to the patient.

Neuro-critical care is a relatively newly recognised and emerging area of sub-speciality care, although it has been a recognised speciality for training and accreditation in the USA since the early 2000's. Its origins come from critical care units developing within neuroscience centres, to care initially for severe traumatic brain injury (STBI), subarachnoid haemorrhage (SAH) and postoperative neurosurgical patients. Most have now expanded to also include both neurological and neurosurgical life-threatening disease such as intra-cerebral haemorrhage (ICH), ischaemic stroke, neuromuscular diseases, refractory status epilepticus and central nervous system infections. There is currently much literature about this subject, with the majority coming from North America. Many of them are single centre studies with historical controls, with few multicentre prospectively controlled studies. There is evidence of the benefits of specialist critical care for the most of the above diseases, which I have briefly summarised in the separate sections below.

\section{SEVERE TRAUMATIC BRAIN INJURY}

Most of the available evidence is within this area of NICU care. Patel's paper ${ }^{[5]}$ from 2005 based on Trauma

This is an open access article distributed under the terms of the Creative Commons Attribution-NonCommercial-ShareAlike 3.0 License, which allows others to remix, tweak, and build upon the work non-commercially, as long as the author is credited and the new creations are licensed under the identical terms.

For reprints contact: reprints@medknow.com

How to cite this article: Tweedie I. Neuro-critical care versus general critical care for neurological injury: Beneficial evidence. J Neuroanaesthesiol Crit Care 2016;3:62-5. 
Audit and Research Network (TARN) data strongly suggested that outcomes for patients managed in a general hospital ICU were worse than those cared for in a neuroscience centre. Such treatment was associated with a $26 \%$ increase in mortality and a 2.15 -fold increase (95\% CI: 1.77-2.60) in the odds of death adjusted for case mix compared with patients treated at a neurosurgical centre. This lead to the National Institute for Clinical Excellence (NICE) modifying their guidance ${ }^{[6]}$ with the following recommendation about management in a neuroscience centre: "Transfer would benefit all patients with serious head injuries (GCS $\leq 8)$, irrespective of the need for neurosurgery". The question mark at the time was around selectivity of the neuroscience units, i.e., were they only taking those patients whom they thought had the chance of a good outcome. After the TARN paper and NICE guidance, the change in the pattern of acceptance by neuroscience centres was noticeable, which if the question above was true should have diluted the better outcomes in the neuroscience centres. However, in 2013, the risk adjustment in neuro-critical care (RAIN) study, ${ }^{[7]}$ a large multicentre observational study run by the Intensive Care, Audit and Research Centre (ICNARC), confirmed better outcomes in neuroscience associated critical care units. Although it did suggest that care was more cost-effective in a single speciality NICU compared to a mixed general/NICU, this was not statistically significant. Interestingly, in those aged over seventy, the outcomes were no better and, in fact, there was a suggestion of greater risk of death if transferred to a specialist unit. The reasons for the apparent better outcomes are not clear although better adherence to protocols is one suggestion with evidence that the use of protocols does improve outcome. ${ }^{[8]}$

Thus, the evidence for traumatic brain injury certainly supports the benefit of specialist neuro-critical care, the possible reasons for which are summarised in Smith's "points of view" paper. ${ }^{[9]}$

\section{INTRA-CEREBRAL HAEMORRHAGE}

$\mathrm{ICH}$ is a devastating disease with poor outcomes and a mortality rate of up to $44 \%$, increasing by a further $50 \%$ by 1 year. As far back as 2001 Diringer and Edwards ${ }^{[10]}$ demonstrated better outcomes for this group of patients when cared for in a specialist critical care unit, with an odds ratio of greater mortality in a general ICU of 3.4. Mirski et al..$^{[11]}$ reported similar findings along with shorter hospital stays and lower costs. Unfortunately, only mortality was quoted and not functional outcome. More recently Damian et al.$^{[12]}$ used ICNARC data to investigate ICU mortality after ICH (10,313 cases) and found that the death rate in a specialist unit was lower, although the length of stay was longer. However, the speed with which the death rate improved in those units was also faster than those seen in general units. Studies such as a second surgical trial in lobar $\mathrm{ICH}^{[13]}$ have not shown any clear therapeutic intervention to have altered the outcome, which suggests the findings of better outcomes in specialist units may be due to the brain centred holistic care given in such units. Rincon and Mayer ${ }^{[14]}$ also suggested that there might be less therapeutic nihilism in specialist ICUs thus negating the self-fulfilling prophecy that little support for these patients brings.

\section{SUBARACHNOID HAEMORRHAGE}

It is clear that the overall management of aneurysmal SAH requires a multi-disciplinary team of which the critical care team is a part. Many treatment modalities have not conclusively been shown to improve outcome apart from nimodipine and securing the aneurysm. As to the latter, we have seen many changes recently with endovascular management replacing surgical clipping in many centres around the world due to the belief that outcomes are better. Although the surgical morbidity is much less, it is certainly our experience that these patients still need very close monitoring and care in a critical care environment to optimise their outcome in light of the risks of delayed ischaemic neurological deficits.

Samuels et al. ${ }^{[15]}$ demonstrated that the presence of neuro-intensivists improved the hospital discharge destination of these patients, such that $36.5 \%$ went home versus $25.2 \%$ of those cared for in a GICU, and they were less likely to need admission to a rehabilitation facility. $\mathrm{McNeill}$ et al. have suggested increased volume of work as a cause for improved outcomes for $\mathrm{SAH}^{,{ }^{[16]}}$ however, Chang's observational study ${ }^{[17]}$ showed that there was no apparent difference in the outcomes between a high volume and low volume unit. Thus, they proposed that it is the expertise of the staff rather than familiarity with the care that is important.

\section{OTHER NEUROLOGICAL DISEASES}

There is some evidence for the effect of neuro-critical care on diseases such as ischaemic stroke, status epilepticus, Guillain-Barre and myasthenia gravis. However, apart from ischaemic stroke, the evidence for better outcomes in a specialist ICU for these types of neurological problems is less convincing. ${ }^{[12]}$ This may relate to the fact that for these diseases the primary critical care issue may be respiratory failure rather a condition that requires aggressive, intensive and specialised interventions. No evidence was found for super-refractory status epilepticus, but I would predict better outcomes in a neuro-centre ICU provided the referral is made early. If true, this would be due to the greater ability to monitor and interpret electroencephalogram continuously with immediate input from Epileptologists, as well as the multidisciplinary Neuro-ICU team. 
It has long been accepted that the management of ischaemic stroke patients in a dedicated 'stroke unit' leads to better outcomes. However, with the increasing use of aggressive interventions such as decompressive craniectomy for malignant middle cerebral artery syndrome and endovascular thrombectomy it is likely that we will see more of these patients admitted to ICUs within neuroscience units. Historical data ${ }^{[18]}$ suggest that their outcomes may be better in an NICU setting.

\section{SUMMARY}

Although much of the above does suggest better outcomes for patients cared for in critical care units associated with neuroscience centres, there are caveats, which must be considered when looking at many of the studies. Although the main UK studies are multi-centre with large patient numbers, the North American experience is less so and may have some explanation in the remuneration system there. Thus, it is important to be aware of the following points when considering the data:

- Many are small single unit studies, and so there may be concerns of bias

- Many are retrospective studies and not adjusted for confounders

- In many the controls are historical

- Neuroscience-units may not be accepting those most likely to die, thus skewing the results.

If we accept that the outcomes are better, the reasons for this remain less clear. Most authors have attempted to find evidence or postulate as to why this may be true. Suare ${ }^{[19]}$ reviews this with specific regard to advances of monitoring and treatments used and intensive staffing, with the conclusion that when all these are brought together, it leads to better outcomes. The following are the arguments put forward, some of which are supported by evidence presented above:

- Specific experience of the multidisciplinary team-leading to earlier recognition of neurological deterioration

- Larger patient numbers, meaning more experience for the whole team

- Brain centred care, i.e., greater awareness by the multidisciplinary team about the relationship of the injured brain and systemic organs

- Greater adherence to brain protective protocols

- Greater use of intensive brain monitoring techniques

- Rapid access to neurosurgical input, imaging and specialist operating theatres

- Early input of specialist physiotherapy and rehabilitation

- A less nihilistic attitude that comes with wider experience

- A more conservative approach to the end of life.
Many of the studies contain a heterogeneous mix of conditions with different emphasis on which aspects of care were reviewed. Thus, although Kramer and Zygun ${ }^{[3]}$ present a review of a large number of heterogeneous cases $(>25,000)$ that strongly supports the case for better outcomes, it still leaves the question as to why. They noted that evidence of the effect of a neurointensivist's input was associated with better mortality and outcome. The perceived benefits of that alone may be reduced the length of stay, cost savings, fewer shunts in SAH patients, improved documentation and increased organ and tissue donation rates.

\section{CONCLUSION}

There is no question that critical care has changed the outcomes in patients with acute neurological problems. ${ }^{[20]}$ There is also convincing evidence that patients with brain injury are better cared for and have better outcomes both in terms of mortality and neurological recovery in critical care units associated with a neuroscience unit as opposed to a general unit in a nonspecialist hospital. However, the evidence that the outcomes are better in a single speciality NICU is not as clear. There is also evidence that the introduction of intensivists with a specific interest in neurological injury may affect outcomes and length of stay positively.

The model of critical care for these patients clearly favours the ICU with direct and dedicated input from a multidisciplinary team that is expert in and familiar with the care required for this group of patients. However, the UK experience does not show a significant difference between the GICU associated with a neuroscience department and a single speciality NICU in terms of outcome. ${ }^{[7]}$ It is suggestive for STBI but not conclusive. When you take into account that in some parts of the world recruitment of intensivists to single speciality critical care can be problematic, is an NICU 'pod' staffed by a multidisciplinary team who are familiar with the problems of neurological injury, within a large GICU co-located with a neuroscience centre the optimal model? ${ }^{[21]}$

\section{Financial support and sponsorship}

Nil.

\section{Conflicts of interest}

There are no conflicts of interest.

\section{REFERENCES}

1. Smith M, Menon D. Neurocritical care. In: GPICS 2015. London: ICS and FICM; 2015.

2. Smith M. I. Neurocritical care: Has it come of age? Br J Anaesth 2004;93:753-5.

3. Kramer AH, Zygun DA. Do neurocritical care units save lives? Measuring the impact of specialized ICUs. Neurocrit Care 2011;14:329-33. 
4. Kramer AH, Zygun DA. Neurocritical care: Why does it make a difference? Curr Opin Crit Care 2014;20:174-81.

5. Patel HC, Bouamra O, Woodford M, King AT, Yates DW, Lecky FE; Trauma Audit and Research Network. Trends in head injury outcome from 1989 to 2003 and the effect of neurosurgical care: An observational study. Lancet 2005;366:1538-44.

6. National Institute for Clinical Excellence. Guidance CG56. Head Injury: Triage, Assessment, Investigation and Early Management of Head Injury in Infants, Children and Adults (Updated version 2014); 2007. Available from: https://www. nice.org.uk/guidance/cg176.

7. Harrison DA, Prabhu G, Grieve R, Harvey SE, Sadique MZ, Gomes $\mathrm{M}$, et al. Risk adjustment in neurocritical care (RAIN) - Prospective validation of risk prediction models for adult patients with acute traumatic brain injury to use to evaluate the optimum location and comparative costs of neurocritical care: A cohort study. Health Technol Assess 2013;17:vii-viii, 1-350.

8. English SW, Turgeon AF, Owen E, Doucette S, Pagliarello G, McIntyre L. Protocol management of severe traumatic brain injury in intensive care units: A systematic review. Neurocrit Care 2013;18:131-42.

9. Teig M, Smith M. Where should patients with severe traumatic brain injury be managed? All patient should be managed in a neurocritical care unit. J Neurosurg Anesthesiol 2010;22:357-9.

10. Diringer MN, Edwards DF. Admission to a neurologic/ neurosurgical intensive care unit is associated with reduced mortality rate after intracerebral hemorrhage. Crit Care Med 2001;29:635-40.

11. Mirski MA, Chang CW, Cowan R. Impact of a neuroscience intensive care unit on neurosurgical patient outcomes and cost of care: Evidence-based support for an intensivist-directed specialty ICU model of care. J Neurosurg Anesthesiol 2001;13:83-92.

12. Damian MS, Ben-Shlomo Y, Howard R, Bellotti T, Harrison D, Griggs $\mathrm{K}$, et al. The effect of secular trends and specialist neurocritical care on mortality for patients with intracerebral haemorrhage, myasthenia gravis and Guillain-Barré syndrome admitted to critical care: An analysis of the Intensive Care National Audit \& Research Centre (ICNARC) national United Kingdom database. Intensive Care Med 2013;39:1405-12.

13. Mendelow AD, Gregson BA, Rowan EN, Murray GD, Gholkar A, Mitchell PM; STICH II Investigators. Early surgery versus initial conservative treatment in patients with spontaneous supratentorial lobar intracerebral haematomas (STICH II): A randomised trial. Lancet 2013;382:397-408.

14. Rincon F, Mayer SA. Neurocritical care: A distinct discipline? Curr Opin Crit Care 2007;13:115-21.

15. Samuels O, Webb A, Culler S, Martin K, Barrow D. Impact of a dedicated neurocritical care team in treating patients with aneurysmal subarachnoid hemorrhage. Neurocrit Care 2011;14:334-40.

16. McNeill L, English SW, Borg N, Matta BF, Menon DK. Effects of institutional caseload of subarachnoid hemorrhage on mortality: A secondary analysis of administrative data. Stroke 2013;44:647-52.

17. Chang TR, Kowalski RG, Carhuapoma JR, Tamargo RJ, Naval NS. Impact of case volume on aneurysmal subarachnoid hemorrhage outcomes. J Crit Care 2015;30:469-72.

18. Bershad EM, Feen ES, Hernandez OH, Suri MF, Suarez JI. Impact of a specialized neurointensive care team on outcomes of critically ill acute ischemic stroke patients. Neurocrit Care 2008;9:287-92.

19. Suarez JI. Outcome in neurocritical care: Advances in monitoring and treatment and effect of a specialized neurocritical care team. Crit Care Med 2006;34 9 Suppl: S232-8.

20. Kramer AH, Zygun DA. Declining mortality in neurocritical care patients: A cohort study in Southern Alberta over eleven years. Can J Anaesth 2013;60:966-75.

21. Tweedie IE, Andrzejowski J. Neurocritical care. Crit Eye 2015:16-7. 